\title{
DIFFERENCES OF SITUATIONAL AND MOTOR ABILITIES IN DEFENSE AT THE WORLD JUNIOR HANDBALL CHAMPIONSHIP
}

\author{
Sanja Bajgorić ${ }^{1}$ Muris Đug ${ }^{2}$ \\ 1 University Study of Health Care, Džemal Bijedić University in Mostar \\ ${ }^{2}$ Faculty of Physical Education and Sport, University of Tuzla
}

Original scientific paper

\begin{abstract}
The research was conducted in order to determine the differences in situational-motor abilities in the defense of men's junior national teams at the 2013 World Handball Championship. Situational - motor abilities in the defense of the national team of Bosnia and Herzegovina in relation to the national teams of Croatia, France, Sweden and Spain were analyzed. The following variables were used to determine differences in defense: SKODEKUK / PR - total - average number of jumps in defense of the whole team, SKODUK / PR1 - total - average number of jumps in defense first player of defense, SKODUK / PR2 - total - average number of jumps in defense second defensive player, SKODUK / PR3 - total - average number of defensive jumps third defensive player, SKODUK / PR4 - total - average number of defensive jumps fourth defensive player, SKODUK / PR5 - total - average number of defensive jumps fifth defensive player and SKODUK / PR6 - total - average number of jumps in the sixth defense player. To determine the partial quantitative differences of variables, which measure the total and average number of attempts to perform an element in the area of situational - motor abilities in defense, we used the mean difference of mean (hereinafter MD), which calculated the average values of selected variables. The average values of variables for the assessment of situational - motor abilities in defense were also calculated. The variable SKODUK / PR4 - total - average number of jumps in the defense of the fourth player shows a statistically significant negative difference in the jumps in the defense of the fourth player between the fourth player in the defense of Bosnia and Herzegovina and the fourth player in the defense of Croatia and Sweden. In the variable SKODUK / PR6 - total - average number of jumps in the defense of the sixth defense player, a statistically significant negative difference can be seen between the sixth player in the defense of the national team of Bosnia and Herzegovina and the sixth player in the defense of Sweden.
\end{abstract}

Key words: handball, juniors, World Championship, defense, situational - motor abilities

\section{INTRODUCTION}

A handball match is a confrontation of two opponents, two teams, which are determined separately by the level of abilities, characteristics and knowledge used to achieve the best result, which means that the confrontation of two opponents produces similar but never the same development or outcome of the match. (Vuleta et al., 2005).

Statistically speaking, the total average effective playing time in handball matches is 48-50 minutes, and the player runs $4-5,6 \mathrm{~km}$ during the match, of which $1-2 \%$ with the ball. The maximum intensity is played about $10 \%$, the submaximal about $46.35 \%$, and the medium intensity about $35.6 \%$ of the total duration of the match. During the game, the player changes the tempo an average of 190 times and performs 16 - 28 maximal jumps (Kovač \& Đukić 1980; Kuchenbecker \& Zieschang, 1992). Also, in the papers (Šibila, et al 2004) the data collected at the matches of the Spanish national handball team were processed. The following results are highlighted, where players make an average of 190 changes of rhythm, 279 changes of direction, 16 jumps during one game.

The handball game records progressive development and constantly requires an intense fight for the ball, a fight with time and space. Increasing the speed and dynamism both in the attack phase and in the defense phase, the speed of performing elements of the game with and without the ball, performing fast actions in the attack, is a characteristic of modern handball.

\section{RESEARCH METHODOLOGY Method of data collection}

The data needed for analysis in this study were collected by observing handball matches at the 2013 World Junior Handball Championship in Bosnia and Herzegovina, and based on official EHF statistics kept during the World Championship. 


\section{Sample of respondents - entities}

The sample of respondents - entities in this research are handball players, juniors - players of five national teams participating in the World Handball Championship for juniors in 2013. Four first-placed national teams (Sweden, Spain, France and Croatia) were analyzed, as well as the fourteenth-ranked Bosnia and Herzegovina national team.

The chronological age of the respondents is from 18 to 21 years, and the survey covered only those respondents who actively participated as players in the matches at this World Cup.

Sample variables

For the purposes of this research, a total of 14 variables were used to assess situational-motor abilities in defense: total number of jumps in defense of the whole team (SKODEKUK), average number of jumps in defense of the whole team (SKODEKPR), total number of jumps in defense ), average number of jumps in defense of the first player of defense (SKODPR1), total number of jumps in defense of second player of defense (SKODUK2), average number of jumps in defense of second player of defense (SKODPR2), total number of jumps in defense of third player of defense (SKODUK3), average number of defensive jumps third defense player (SKODPR3), total number of defensive jumps fourth defense player (SKODUK4), average number of defensive jumps fourth defense player (SKODPR4), total number of defensive jumps fifth defense player (SKODUK5), average number jumps in defense of the fifth player of defense (SKODPR5), total number of jumps in defense of the sixth player of defense (SKODUK6), average number of jumps in defense of the sixth player of defense (SKODPR6).

\section{Data processing methods}

To determine the partial quantitative differences of variables, which measure the total and average number of attempts to perform an element in the area of situational - motor abilities in defense, we used the mean difference of mean (hereinafter $M D$ ), which calculated the average values of selected variables. The standard classification of differences, derived from percentile calculations, implies the following effect limits: 0-0.3 no effect (difference), up to 0.5 is a small difference, up to 0.8 is medium difference and over 0.8 is large difference. As it is known that in $95 \%$ of cases the values of MD will be less than 1.7, such values can be considered statistically significant differences. The direction of the difference can be positive or negative. Also, average values for the assessment of situational - motor abilities in defense were calculated. The statistical program SPSS and the program Microsoft Excell were used for data processing.

\section{RESULTS AND DISCUSSION}

Table 1 analyzes the average values of variables for the assessment of situational - motor abilities in defense. In the variable SKODEKPR - average number of jumps in defense, the entire team, on average, the Croatian national team had the largest number of jumps in defense. On average, the national teams of Sweden, Bosnia and Herzegovina and Spain had a less numberof jumps in defense, while the national team of France had the lowest number of jumps in defense. Analysis of the value of results for the variable SKODPR1 - average number of jumps in the defense of the first player of defense, it is seen that the first players in the defense of all analyzed teams had an average less number of jumps. In the variable SKODPR2 average number of jumps in defense, the second defensive player, it can be seen that the second player in the defense of the Croatian national team had the most jumps in defense on average. On average, the second defense player of the national team of Bosnia and Herzegovina had a less number of jumps in defense, while on average the second number of defensive jumps had the second defense player of the national teams of France, Sweden and Spain. Analysis of the value of results for the variable SKODPR3 - average number of jumps in the defense of the third defense player, shows that on average the highest number of jumps had third players in the defense of Croatia, Spain, Bosnia and Herzegovina. On average, the third players in the defense of the national teams of France and Sweden had a less number of jumps. In the variable SKODPR4 - average number of jumps in defense, the fourth player in defense, it can be seen that the fourth player of the Swedish national team had the most jumps in defense on average. On average, the fourth player in the defense of the Croatian national team had a less number of jumps than the four players in the defense of the Swedish national team, while the fourth players in the defense of the national team of France, Spain and Bosnia and Herzegovina had the least number of jumps. An analysis of the values for the variable SKODPR5 the average number of jumps in defense by the fifth player of the defense, shows that on average the fifth player of the Swedish national team had the most jumps in defense. On average, the fifth player of the Croatian national team had a less number of jumps in defense, while the fifth players 
of the national teams of France, Bosnia and Herzegovina and Spain had the least number of jumps in defense. The analysis of the value of the results for the variable SKODPR6 - the average number of jumps in the defense of the sixth player of the defense, low values and a less number of jumps in the defense of the sixth player in the defense of the analyzed teams.

Table 1. Average values of variables for the assessment of situational - motor abilities in the defense of average - analyzed representations

\begin{tabular}{|l|l|l|l|l|l|}
\hline Varijable & Croatia & France & Bosna Herzegovina & Sweden & Spain \\
\hline SKODEKPR & 74,333 & 46,333 & 53,667 & 61,000 & 53,333 \\
\hline SKODPR1 & 1,667 & 1,667 & 2,000 & 1,000 & 1,000 \\
\hline SKODPR2 & 15,000 & 7,667 & 10,333 & 5,667 & 6,333 \\
\hline SKODPR3 & 25,000 & 14,667 & 21,333 & 16,667 & 20,667 \\
\hline SKODPR4 & 21,667 & 14,333 & 13,333 & 23,667 & 17,000 \\
\hline SKODPR5 & 9,667 & 6,000 & 6,667 & 12,333 & 7,333 \\
\hline SKODPR6 & 0,667 & 1,333 & 0,000 & 1,667 & 1,000 \\
\hline
\end{tabular}

Table 2 analyzes the variables for assessing situational - motor abilities in defense. Analysis of the value of differences for the variable SKODEKUK / PR - it is seen that there is no difference between the national teams of Bosnia and Herzegovina, France and Spain. There is a small negative difference between the national teams of Bosnia and Herzegovina and Sweden (the national team of Bosnia and Herzegovina had slightly fewer jumps in defense than the national team of Sweden). There is a big negative difference between the national teams of Bosnia and Herzegovina and Croatia (the Croatian national team had more jumps in defense than the national team of Bosnia and Herzegovina - a big difference in jumps). Analyzing the value of differences for the variable SKODUK / PR1 - there are no differences between the first player in the defense of the national team of Bosnia and Herzegovina and the first players in the defense of the national team of Croatia and France. There is a medium positive difference between the first player in the defense of the national team of Bosnia and Herzegovina and the first player in the defense of the national team of Sweden and Spain. Analyzing the value of differences for the variable SKODUK / PR2 - there is a medium positive difference between the second player in the defense of the national team of Bosnia and Herzegovina and the second player of the French national team. There is a medium negative difference between the second player of the national team of Bosnia and Herzegovina and the second player of the Croatian national team. There is also a big positive difference between the second player in the defense of the national team of Bosnia and Herzegovina and other players in the defense of the national team of Sweden and Spain (the second player in the defense of the national team of Bosnia and
Herzegovina made more jumps in defense - a big difference). By analyzing the value of the differences for the variable SKODUK / PR3 - there is no difference between the third player in the defense of the national team of Bosnia and Herzegovina and the third player in the defense of the Swedish national team. There is a small negative difference in the jumps in defense between the third player in the defense of the national team of Bosnia and Herzegovina and the third player in the defense of the Croatian national team. There is a medium positive difference between the third player in the defense of the national team of Bosnia and Herzegovina and the third player in the defense of the national team of France and Sweden. By analyzing the value of the differences for the variable SKODUK / PR4 - there are no differences in jumps in defense between the fourth player in the defense of the national team of Bosnia and Herzegovina and France. There is a medium negative difference between the fourth player in the defense of the national team of Bosnia and Herzegovina and Spain (which means that the fourth player in the defense of the national team of Bosnia and Herzegovina made medium fewer jumps than the fourth player in the defense of the Spanish national team). There is a statistically significant negative difference between the fourth player in the defense of the national team of Bosnia and Herzegovina and the fourth player in the defense of the national team of Croatia and Sweden. Analyzing the values of the differences for the variable SKODUK / PR5 - there are no differences in jumps in defense between the fifth player of the national team of Bosnia and Herzegovina and the five players in the defense of the national teams of Croatia, France and Spain. There is a medium negative difference between the 
fifth player in the defense of the national team of Bosnia and Herzegovina and the fifth player in the defense of the Swedish national team. Analyzing the value of differences for the variable SKODUK / PR6 - there is a medium negative difference between the sixth player in the defense of the national team of Bosnia and Herzegovina and the sixth player in the defense of the Croatian national team. There is a big negative difference between the sixth player in the defense of the national team of Bosnia and Herzegovina and the sixth player in the defense of the national team of France and Spain. There is a statistically significant negative difference between the sixth player in the defense of the national team of Bosnia and Herzegovina and the sixth player in the defense of the Swedish national team.

Table 2. MD analysis of variables for the assessment of situational - motor abilities in defense as a team and individually by playing positions of the national team of Bosnia and Herzegovina in relation to the national team of Croatia, France, Sweden and Spain

\begin{tabular}{|l|l|l|l|l|l|l|l|}
\hline NATIONAL TEAMS & $\begin{array}{l}\text { SKODEK } \\
\text { UK/PR }\end{array}$ & $\begin{array}{l}\text { SKOD } \\
\text { UK/PR1 }\end{array}$ & $\begin{array}{l}\text { SKOD } \\
\text { UK/PR2 }\end{array}$ & $\begin{array}{l}\text { SKOD } \\
\text { UK/PR3 }\end{array}$ & $\begin{array}{l}\text { SKOD } \\
\text { UK/PR4 }\end{array}$ & $\begin{array}{l}\text { SKOD } \\
\text { UK/PR5 }\end{array}$ & SKOD \\
\hline BIH-CRO & $-0,840$ & 0,150 & $-0,648$ & $-0,388$ & $-2,182$ & $-0,297$ & $-0,653$ \\
\hline BIH-FRA & 0,286 & 0,131 & 0,574 & 0,648 & $-0,215$ & 0,068 & $-1,306$ \\
\hline BIH-SVE & $-0,302$ & 0,506 & 1,191 & 0,500 & $-2,146$ & $-0,559$ & $-3,266$ \\
\hline BIH-SPA & 0,013 & 0,506 & 1,020 & 0,073 & $-0,753$ & $-0,062$ & $-1,131$ \\
\hline
\end{tabular}

\section{CONCLUSION}

The main goal of this study was to determine the differences between situational and motor abilities in the defense of the national team of Bosnia and Herzegovina in relation to the national teams of Croatia, France, Sweden and Spain. The following variables were used to determine differences in defense: SKODEKUK / PR - total - average number of jumps in defense of the whole team, SKODUK / PR1 - total - average number of jumps in defense first player of defense, SKODUK / PR2 - total average number of jumps in defense second defensive player, SKODUK / PR3 - total - average number of defensive jumps third defensive player, SKODUK / PR4 - total - average number of defensive jumps fourth defensive player, SKODUK / PR5 - total - average number of defensive jumps fifth defensive player and SKODUK / PR6 - total average number of jumps in the sixth defense player.

\section{REFERENCES}

1. Bajgorić, S. (2016). Valorization and levels of differences in situational-motor abilities and situational efficiency of teams at the World Junior Handball Championship in 2013. Doctoral thesis. Faculty of Physical Education and Sports. Tuzla

2. Kovac, J., Djukic, M. (1980). Quantitative analysis of handball players' motor activities in competitive conditions. Sports practice. Belgrade.

3. Kuchenbecker, R., Zieschang, K. (1992). Training analysis in handball and training for training planning.

4. Šibila, M., Vuleta, D., Pori, P. (2004). Position - related differences in volume and intensity of large - scale cyclic movements of male players in handball. Kinesiology (36) 2004 1: 58-68.

5. Vuleta, D., Milanović, D., Gruić, I., \& Ohnjec, K. (2005). Influence of the goals scored on the final outcomes of the matches of the 2003 World Handball Championships for Men in Portugal.

\section{Correspondence to.}

Sanja Bajgorić

Email: sbajgoric@yahoo.it
The national teams of Bosnia and Herzegovina, France and Spain had the same effect in terms of the number of jumps in defense. Sweden's national France's slightly weaker performance, while Croatia was the best of all. In terms of playing positions in defense, the first and sixth players of Bosnia and Herzegovina stand out because the first player was slightly to medium better than all the . When way through a large number of variables, then two aspects must be taken: national teams had a different game set, so the effects of entire teams differ individually by playing positions, or if the slaces, had a similar game setup but the effect is on such a playing place was better / worse. 